\title{
Infrared Spectra of D-Talose Monobenzoate and Related Substances
}

\author{
H. S. Isbell, J. E. Stewart, H. L. Frush, J. D. Moyer, and F. A. Smith
}

\begin{abstract}
Infrared absorption spectra for D-talose monobenzoate, $\alpha$-D-talose, $\beta$-D-talose, pentaacetyl- $\alpha$-D-talose, tetraacetyl- $\alpha$-D-talose, and $\beta$-L-rhamnose 1,2-(methyl orthoacetate) show that the previously held, orthoacid structure for $\mathrm{D}$-talose monobenzoate is not correct. The spectrum and the method of synthesis of D-talose monobenzoate indicate that the compound is a 1-benzoyl-D-talopyranose. The infrared absorption of the alpha and beta pyranose modifications of D-talose was found to be anomalous in that each form shows absorption heretofore considered characteristic of the alpha modification as well as that considered characteristic of the beta.
\end{abstract}

\section{Discussion}

By the action of perbenzoic acid on D-galactal (D-talal), Pigman and Isbell [1] ${ }^{2}$ obtained a D-talose monobenzoate for which they suggested an orthoester structure because of its unusual properties. The substance showed a rapid, complex mutarotation in methanol, a similar but somewhat slower mutarotation in pyridine, and almost no mutarotation in dilute hydrochloric acid. In light of the properties of certain orthobenzoates of ribose recently described by Fletcher and Ness [2], it seemed desirable to reinvestigate the structure of the talose derivative.

A sample of the product prepared by Pigman and Isbell in 1937 was available, but had turned slightly brown. After two recrystallizations from dimethoxyethane, the melting point was sharper and higher than that reported earlier, and the mutarotation in methanol followed the same course, but at a slightly lower rate. The recrystallized compound, like the original, did not yield a phenylhydrazone; hence the reducing group must be blocked by the benzoyl group, which could be present either as the normal ester (I) or as the orthoester (II). However, only the normal ester would show infrared absorption characteristic of a carbonyl group.

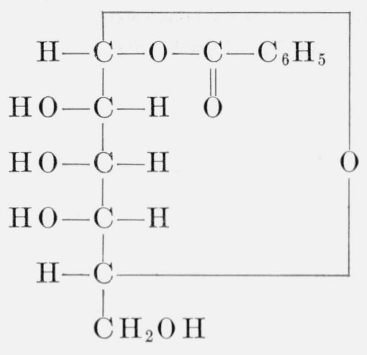

I

D-Talopyranose 1-benzoate.

1 This work was sponsored by the Chemistry Branch of the Office of Naval Research, Department of the Navy, Washington, D. C.

${ }_{2}$ Figures in brackets indicate the literature references at the end of this paper.

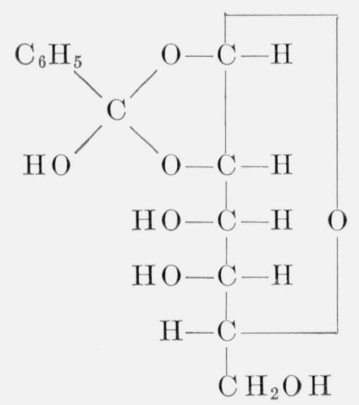

II

$\beta$-D-Talose 1,2-(orthobenzoic acid).

It has now been found that the infrared absorption spectrum of the original D-talose monobenzoate (fig. 1 ), which is identical with the spectrum of the recently repurified product, shows a strong absorption at $5.77 \mu$, characteristic of the ester carbonyl group. In contrast, $\beta$-L-rhamnose 1,2-(methyl orthoacetate), which has been characterized as an orthoester by means of its ultraviolet absorption spectrum $[3,4]$, shows no absorption near this wavelength. Thus, it can be concluded that the D-talose monobenzoate is not an orthoester but a normal benzoic ester possessing a carbonyl group. This conclusion is also supported by the observed absorption at $7.93 \mu$, because normal benzoates show characteristic absorption in this region $[5,6]$. The lack of formation of a hydrazone, in conjunction with the infrared absorption, requires that the product be a D-talose 1-benzoate.

It seemed possible that a study of infrared absorp tion might aid in assigning alpha or beta configuration to the D-talose 1-benzoate. A study of the spectra of over 75 hexoses and substituted hexoses had previously shown that the alpha anomers of the more common configurations have bands in the 11.6- to $12.4-\mu$ region, whereas the beta anomers have bands in the 11.2 - to $11.7-\mu$ region. ${ }^{3}$ These regions

\footnotetext{
3 The spectra will be presented in papers now in preparation.
} 

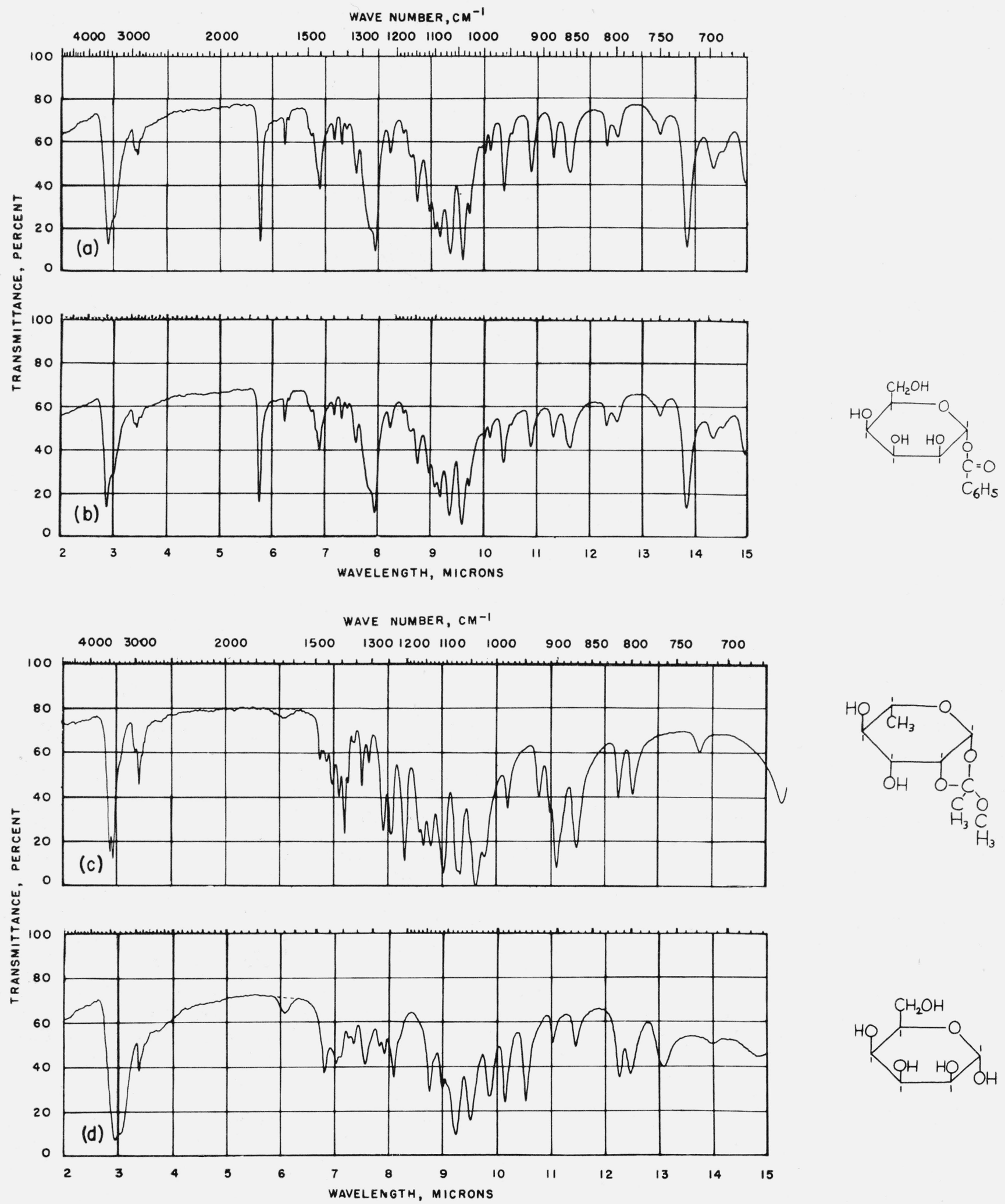

FiguRE 1. Spectrograms of materials in potassium chloride pellets

a, D-Talose monobenzoate (original'sample); b, D-talose monobenzoate (recrystallized); c, $\boldsymbol{\beta}$-L-rhamnose 1.2-(methyl orthoacetate); d, $\boldsymbol{\alpha} \cdot \mathrm{D}$-talopyranose. 

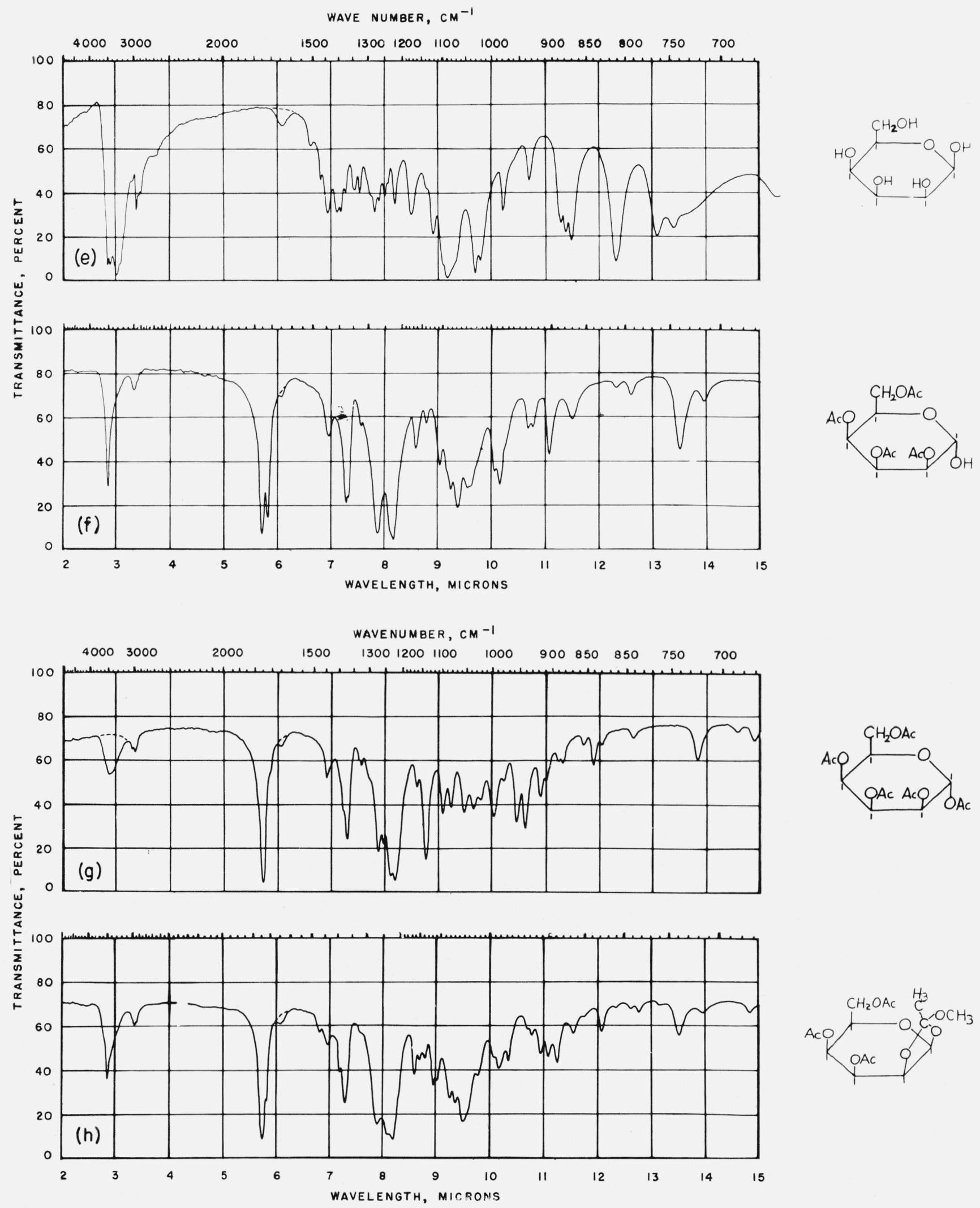

Figure 1. Spectrograms of materials in potassium chloride pellets-Continued

e, $\boldsymbol{\beta}$-D-talopyranose; f, tetraacetyl- $\boldsymbol{\alpha}$-D-talopyranose; g, pentaacetyl- $\alpha$-D-talopyranose; h, triacetyl- $\boldsymbol{\beta}$-D-talose 1,2-(methyl orthoacetate) (old sample). 
are much narrower for specific hexose types. The existence of characteristic infrared absorption for the common hexose types is in agreement with the observations of Barker et al. [7]. It now appears, however, that the infrared absorption of the D-talopyranoses and a number of related derivatives is anomalous in that bands occur in hoth of the regions mentioned (see table 1 and fig. 1).

In light of this fact, one cannot assign an alpha or beta configuration to $\mathrm{D}$-talose 1-benzoate on the basis of absorption in the so-called "alpha and beta regions." It follows also that absorption in these regions cannot be used indiscriminately for the assignment of alpha or beta configuration to all types of pyranose derivatives. Whatever the difference between the absorption found for talose and that found for other hexoses, it is apparent that infrared measurements must be extended to all possible pyranose types before generalizations as to characteristic absorption can be held valid.

TABLE 1. Absorption bands of $\mathrm{D}$-talose derivatives in the 11.2to $12.7-\mu$ region

\begin{tabular}{|c|c|c|}
\hline Compound & Wavelength of bands & $\begin{array}{c}\text { Curve in } \\
\text { fig. } 1\end{array}$ \\
\hline $\begin{array}{l}\text { D-Talose monobenzoate } \\
\alpha \text {-D-Talopyranose } \\
\beta \text {-D-Talopyranose } \\
\text { Tetraacetyl- } \alpha \text {-D-talopyranose- } \\
\text { Pentaacetyl- } \alpha \text {-D-talopyranose } \\
\text { Triacetyl- } \beta \text {-D-talose } 1,2 \text {-(meth- } \\
\text { yl orthoacetate). }\end{array}$ & $\begin{array}{c}\mu \\
11.31,11.63,12.32,12.52 \ldots \ldots \\
11.44,12.25,12.45,12 \ldots \\
11.28,11.38,11.48,12.31 \ldots \\
11.49,12.32,12.59 \\
11.40,11.68,11.87,12.02,12.61 \\
11.23,11.54,12.06,12.31,12.58\end{array}$ & $\begin{array}{l}\mathrm{b} \\
\mathrm{d} \\
\mathrm{e} \\
\mathrm{f} \\
\mathrm{g} \\
\mathrm{h}\end{array}$ \\
\hline
\end{tabular}

Although infrared studies do not distinguish satisfactorily between the alpha and beta anomers of talose derivatives, the mode of synthesis of Dtalose 1-benzoate provides a basis for assigning the configuration at carbon 1 . The compound was prepared by the oxidation of D-galactal (III) with perbenzoic acid. Reaction of ethylenic compounds with perbenzoic acid gives anhydrides by the process clearly described by Waters [8]. Thus D-galactal, on oxidation with perbenzoic acid, forms, as intermediates, 1,2-anhydro-D-talopyranose, IV, and 1,2anhydro-D-galactopyranose, $V$. The products isolated are obtained by cleavage of these 1,2-anhydrides. In accordance with the concept presented by one of us in 1940, cleavage of the anhydrides takes place by a mechanism in which the entering group approaches the face of one of the carbons from the side opposite the ring oxygen and combines with inversion [9]. Reaction of carbon 2 of IV with benzoate ion by an opposite-face mechanism would give $\beta$-D-galactopyranose 2 -benzoate, and reaction of carbon 2 of $\mathrm{V}$ would give $\alpha$-D-talopyranose 2 benzoate. These compounds have not been found. However, reaction of carbon 1 of IV would give $\alpha$-D-talopyranose 1-benzoate $\mathrm{I}$, and reaction of carbon 1 of $\mathrm{V}$ would give $\beta$-D-galactopyranose 1-benzoate. ${ }^{4}$ Hence the talose 1-benzoate found experimentally

${ }^{4}$ By perbenzoic acid oxidation of triacetyl-D-galactal, Levene and Tipson [10] obtained tri- $O$-acetyl- $\beta$-D-galactopyranose 1 -benzoate, the product to be expected by the mechanism indicated here. should have the alpha pyranose structure. This structure does not account for the mutarotation of the substance observed in methanol and in pyridine. Explanation of these unusual properties must await further experimental study.<smiles>CC(CO)C(O)C(O)CO</smiles>

III

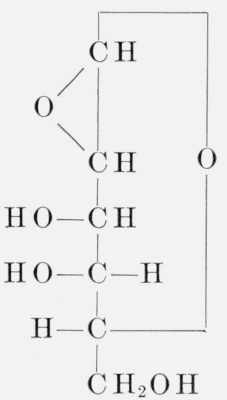

IV
D-Galactal.

1,2-Anhydro-D-talopyranose.<smiles>OCC(CO)C(O)C(O)C1CO1</smiles>

V

1,2-Anhydro-D-galactopyranose.

\section{Experimental Details}

\subsection{D-Talose Monobenzoate}

The product prepared by Pigman and Isbell in 1937 melted at $160^{\circ}$ to $175^{\circ} \mathrm{C}$ in 1955 , and showed the infrared absorption given in curve $a$ of figure 1 . Two recrystallizations from dimethoxyethane changed the melting point to $180^{\circ}$ to $182^{\circ} \mathrm{C}$ without an appreciable change in absorption (curve $b$ ). Hence recrystallization from dimethoxyethane occurred without change in structure. When dissolved in anhydrous methanol, the recrystallized product gave the mutarotation reported in table 2 . The rate of

TABLE 2. Rotation of $\mathrm{D}$-talose monobenzoate in methanol at $20^{\circ} \mathrm{C}$ (0.0512 g made up to $10 \mathrm{ml}$ with anhydrous methanol, and read in a 2-dm tube.)

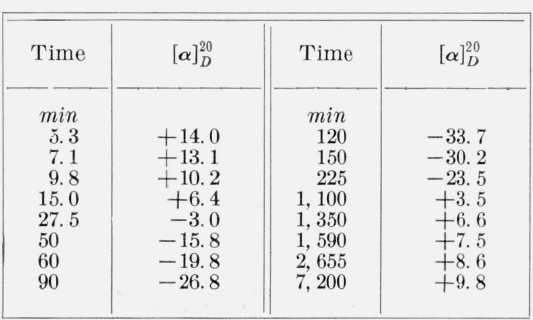


mutarotation is somewhat lower than that observed in 1937, but the over-all change is essentially the same.

\section{2. $\beta$-L-Rhamnose 1,2-(Methyl Orthoacetate)}

This compound was prepared by the method of Fischer, Bergmann, and Rabe [11]. The product, after two recrystallizations from hot ethyl acetate, melted at $143^{\circ}$ to $144^{\circ} \mathrm{C}$, in agreement with the reported value.

\section{3. $\alpha$-D-Talopyranose}

The material used for the spectrogram was obtained by crystallization of the sugar from water with the addition of methanol. The product had a melting point of $131^{\circ}$ to $134^{\circ} \mathrm{C}$, and gave a complex mutarotation like that reported previously [12]. The specific rotation, $[\alpha]_{D}^{20}$, extrapolated to zero time, was $+67^{\circ}$, in substantial agreement with the recorded value of $+68^{\circ}$.

\section{4. $\beta$-D-Talopyranose}

The optical rotation of the $\beta$-D-talopyranose prepared in 1937 showed that the material had changed in large measure to the more stable alpha modification. Hence a new sample was prepared. $\alpha$-DTalose $(15 \mathrm{~g})$ was dissolved in about $50 \mathrm{ml}$ of water. After $18 \mathrm{hr}$, the solution was diluted with $150 \mathrm{ml}$ of absolute ethanol and cooled by immersing in a batb at about $-10^{\circ} \mathrm{C}$. The solution was seeded with 0.5 $\mathrm{g}$ of finely powdered $\alpha$-D-talose, and shaken for 45 min. The crystals that formed were then separated by filtration. On standing at a low temperature, the filtrate yielded a small crop of $\beta$-D-talose. The process was repeated, to obtain a total of about $1 \mathrm{~g}$ of $\beta$-D-talose. The combined crops were then dis. solved in $3 \mathrm{ml}$ of ice-cold water, and $5 \mathrm{ml}$ of cold, absolute ethanol was added; crystallization began immediately. After $1 \mathrm{hr}$, the crop was removed by filtration, washed first with ethanol and then with 2-propanol, and finally thoroughly dried.

The specific rotation, $[\alpha]_{D}^{20}$, of this material, extrapolated to zero time, was $+13.3^{\circ}$, and the melting point was $121^{\circ}$ to $123^{\circ} \mathrm{C}$. The values agree with those previously recorded $[1]\left([\alpha]_{D}^{20}+13.2^{\circ}\right.$ and $\mathrm{mp}$ $120^{\circ}$ to $\left.121^{\circ} \mathrm{C}\right)$.

\subsection{Pentaacetyl- $\alpha$-D-talopyranose}

A sample of the compound previously reported [1] was recrystallized from hot ethanol. The specific rotation, $[\alpha]_{D}^{20}$, of the recrystallized substance was $+70^{\circ}$ and the melting point $106^{\circ}$ to $107^{\circ} \mathrm{C}$, in agreement with the published values.

\subsection{Tetraacetyl- $\alpha$-D-talopyranose}

A sample of tetraacetyl- $\alpha$-D-talose, prepared in 1937, was recrystallized from hot water. The melting point was $114^{\circ}$ to $116^{\circ} \mathrm{C}$ and the initial specific rotation, $[\alpha]_{D}^{20}$, was $+41.5^{\circ}$ in U. S. P. chloroform. The constants differ slightly from those reported $[1]\left([\alpha]_{D}^{20},+42.8^{\circ}\right.$ and $\mathrm{mp} 112^{\circ}$ to $\left.113^{\circ} \mathrm{C}\right)$, but the amount of material available was not sufficient for further study.

\subsection{Triacetyl- $\beta$-D-talose 1,2-(Methyl Orthoacetate)}

The few milligrams of this material remaining from the original preparation of Pigman and Isbell was found to have a melting point of $77^{\circ}$ to $83^{\circ} \mathrm{C}$ (reported, $91.5^{\circ}$ to $92.5^{\circ}$ C). Because of lack of material, the infrared absorption spectrum was determined without prior recrystallization.

\subsection{Measurement of Infrared Absorption}

The spectrograms recorded in figure 1 were made with a Perkin-Elmer model 21 spectrophotometer, using the alkali halide pellet technique. The pellets were prepared from a mixture of $100 \mathrm{mg}$ of potassium chloride and $0.4 \mathrm{mg}$ of the sample. The material was pressed in a 3 -piece die, $9.5 \mathrm{~mm}$ in diameter, patterned after one described by Anderson and Woodall [13]. Pressure was applied with a shop vise. The spectra were run without compensation for reflection or scatter by the pellet.

\section{References}

[1] W. W. Pigman and H. S. Isbell, J. Research NBS 19, 189 (1937) RP1021

[2] H. G. Fletcher, Jr., and R. K. Ness, J. Am. Chem. Soc. ซ⿱, 5337 (1955).

[3] E. Braun, Ber. deut. chem. Ges. 63, 1972 (1930)

[4] E. Pacsu, Advances in Carbohydrate Chem. 1, 107 (1945).

[5] L. J. Bellamy, The infrared spectra of complex molecules, p. 163 (Methuen \& Co., Ltd., London, or J. Wiley \& Sons, Inc., New York, N. Y., 1954)

[6] R. N. Jones, P. Humphries, F. Herling, and K. Dobriner, J. Am. Chem. Soc. 73, 3215 (1951).

[7] S. A. Barker, E. J. Bourne, R. Stephens, and D. H. Whiffen, J. Chem. Soc. 1954, 3468.

[8] W. A. Waters, Gilman's organic chemistry, vol. 4, p. 1165 (J. Wiley \& Sons, Inc., New York, N. Y., 1953).

[9] H. S. Isbell, Ann. Rev. Biochem. 9, 65 (1940).

[10] P. A. Levene and R. S. Tipson, J. Biol. Chem. 93, 631 (1931).

[11] E. Fischer, M. Bergmann, and A. Rabe, Ber. deut. chem. Ges. 53, 2362 (1920).

[12] H. S. Isbell and W. W. Pigman, J. Research NBS 18, 141 (1937) RP969.

[13] D. H. Anderson and N. B. Woodall, Anal. Chem. 25, 1906 (1953).

Washington, April 9, 1956. 